\title{
The Risk Observatory: Developing an Aviation Safety Information Sharing Platform in Europe
}

\author{
Joram Verstraeten*, Gerben van Baren, Rombout Wever \\ Netherlands Aerospace Centre (NLR) \\ Anthony Fokkerweg 2, 1059 CM, Amsterdam, The Netherlands \\ *Tel: +31885113216 \\ *Email: joram.verstraeten@nlr.nl
}

doi:10.5296/jss.v2i2.10443 URL: http://dx.doi.org/10.5296/jss.v2i2.10443

\begin{abstract}
In Europe the accident rate in commercial aviation has stagnated at around 40 accidents per ten million flights: forty times higher than Europe's ambition. Currently safety management is done per organisation, focusing on an organisation's own domain. European research institutions and the aviation sector have joined their expertise in the EU-funded Future Sky Safety Programme. One project within the programme aims to enable inter-organisation and inter-domain safety management. The four year project will deliver a tool, the Risk Observatory, which acquires safety data and translates it into actionable safety information. In the first year, more than 20 European stakeholder organisations have been consulted to express their needs for a Risk Observatory. The resulting requirements have been used to develop an early prototype: mock-ups of dashboards and a user interface. The Risk Observatory has four main elements. (1) Tracking of safety performance indicators distilled from input safety data. (2) Trend diagrams and visualisations of accident risk. Risk models will be developed to translate the input data into accident risk. The risk models also allow (3) assessment of the effects of mitigation measures. There is added value in sharing qualitative safety knowledge, such as identified hazards, therefore, (4) a searchable repository is included. The early prototype is successfully used to validate and further specify the requirements. The need for inter-organisation and inter-domain safety data dissemination was confirmed by the stakeholders. In the coming years the project will develop a fully functional prototype risk observatory, risk models and a business model.
\end{abstract}

Keywords: Aviation safety, safety data sharing, safety risk modelling 


\section{Ml Macrothink}

\section{Towards 1 accident every 10 million flights}

While the fatal accident rate for commercial aviation in Europe has been decreasing over the last decades, the accident rate stagnates at around 40 accidents per ten million flights (Figure 1): forty times higher than Europe's ambition (European Commission, 2011). Aviation organisations introduced safety management systems to get a clearer picture of risks and to focus risk mitigations on the highest risks. However, to date safety management is performed on islands: it is done per organisation, taking into account an organisation's own domain. A breakthrough is needed.

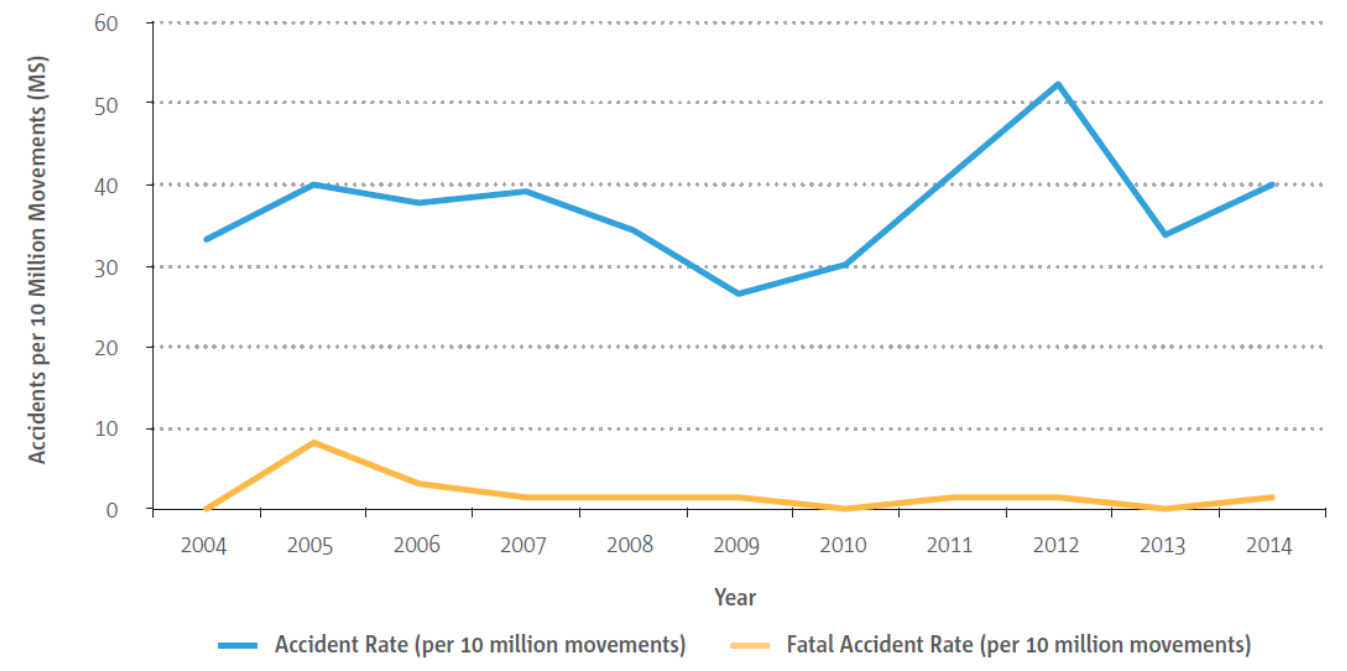

Figure 1. Evolution of the accident rates for EASA Member States (EASA, 2015).

This paper will detail how the breakthrough can be provided by enabling inter-organisation and inter-domain safety management. This is done by sharing safety data and information ${ }^{1}$ between organisations and domains (the following domains are considered: aircraft operators, air navigation service providers, aircraft manufacturers, airports and authorities). Data sharing is already practiced in the nuclear industry (IAEA, 2008) and in the United States aviation sector (FAA, 2016). There is a clear wish to extend this practice throughout the aviation industry, as for example formulated by ICAO (2013) and AustroControl (Hoffmann, 2016). European research institutions, airlines, air navigation service providers, manufacturers and authorities have joined their expertise in the EU-funded Future Sky Safety Programme. One four-year project within the programme started in 2015 to develop a prototype risk observatory aiming to enable inter-organisation and inter-domain safety management.

\section{What is a risk observatory?}

A risk observatory enables improved safety management by facilitating the exchange of safety information across organisations and across domains. The safety information will be used for safety risk management and safety assurance, the core pillars of a safety management system. The risk observatory improves an organisation's ability to perform

\footnotetext{
${ }^{1}$ Please refer to the glossary at the end of the paper for definitions of key terminology used.
} 


\section{Macrothink Institute ${ }^{T M}$}

hazard identification and risk assessment (part of safety risk management) and safety monitoring (part of safety assurance) by giving access to safety information from other organisations and domains. Proper safety risk management and safety assurance facilitates informed decision making to make sure the available resources are used for the biggest possible safety improvement. The foreseen users of the risk observatory are safety analysts, safety managers and accountable managers. For these users the risk observatory is a web-based collection of pages containing searchable and specifiable safety information. To realise such a web-based environment, supporting software and hardware are needed. To run and maintain the risk observatory a dedicated organisation is required.

The pivotal asset of the risk observatory is safety data. It is foreseen that the risk observatory has access to a variety of safety data sources. These sources include mandatory and voluntary occurrence reports, flight data monitoring (FDM) data, radar track data and exposure data (e.g. number of flights and flight hours). Additional contextual data is foreseen, such as weather data and infrastructural data. The key value proposition of the risk observatory is to offer safety information to the user. Safety information is obtained by processing, organising or analysing safety data in a given context so as to make it useful for safety management purposes (ICAO, 2016).

\section{Stakeholder consultations}

Stakeholders were consulted to identify gaps in current safety management practices and to elicit the needs and wishes from future users of the risk observatory. More than 20 organisations have been interviewed throughout Europe. For an overview of the geographical spread of interviewed organisations, refer to Figure 2.

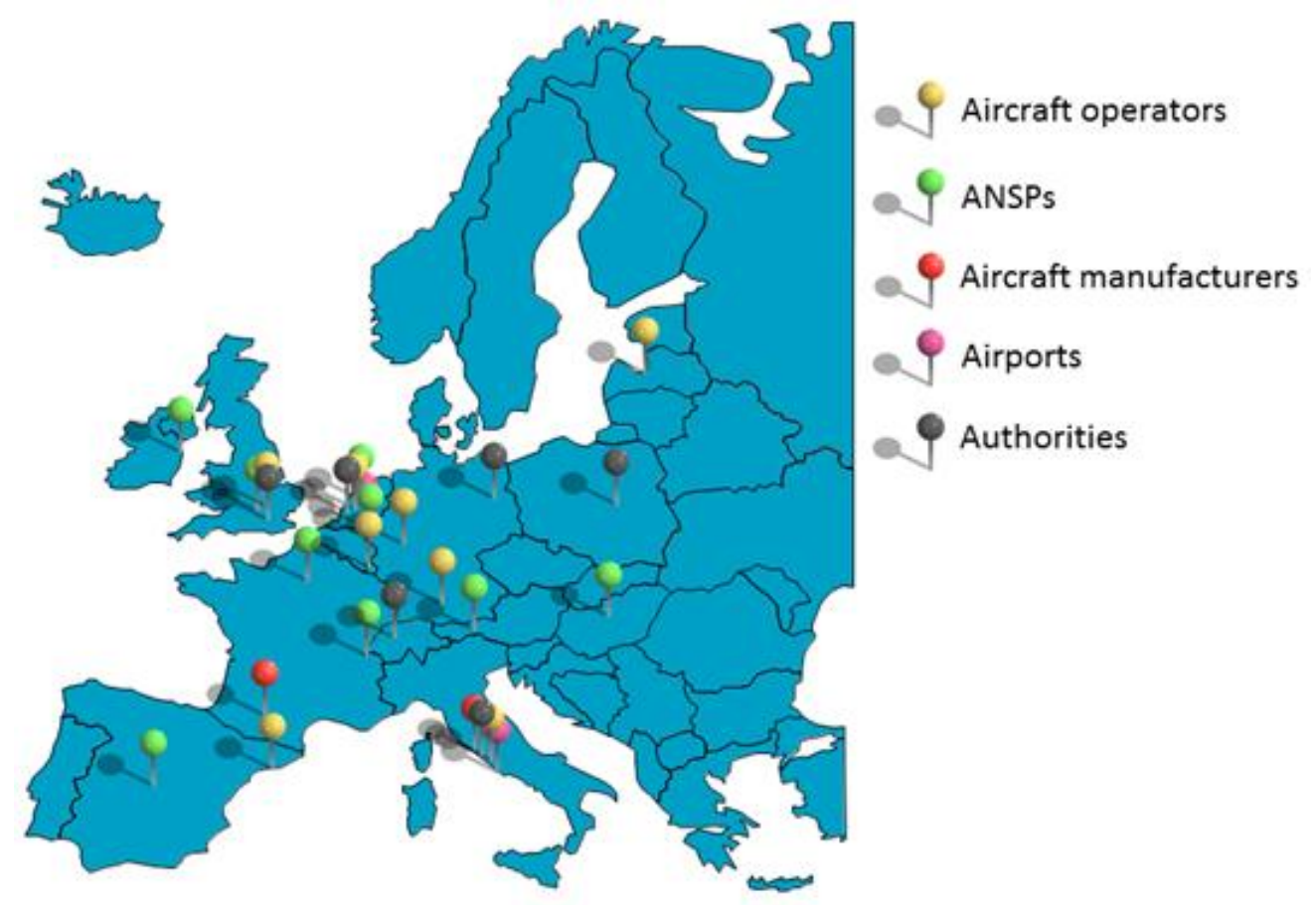

Figure 2. Geographical spread of interviewed organisations. 


\subsection{Gaps in current safety management practices}

For the purpose of this paper two gaps are highlighted.

\subsubsection{Safety data is not used to the full extent}

In aviation a large amount of safety data is produced. Examples are voluntary and mandatory occurrence reports, FDM data and radar track data. In the opinion of the authors this safety data is currently insufficiently used, this is true for data that is produced by an organisation itself, but especially for data that is externally produced. This gap makes it impossible to get a full picture of safety risks which causes uncertainty with regard to current safety performance.

\subsubsection{Processing of safety data and translation to safety information is not done effectively}

In many organisations the available human resources are mostly used for manual processing of safety data and the reactive analysis based on individual occurrences. Organisations feel overloaded with data and are not able to use it for proactive safety management.

\subsection{Needs and wishes of consulted stakeholders}

For the purpose of this paper three needs and wishes are highlighted.

\subsubsection{Provide the opportunity to compare safety performance}

Comparing safety performance using the risk observatory received mixed feedback: for some stakeholders it is a key asset, others are doubtful about its usefulness and desirability. Those in favour are mostly from smaller operators. They welcome the ability to benchmark their performance, lacking sufficient information from their own operation. One operator stated: "I have no idea if our bird strike rate is high or low". It is foreseen that the risk observatory offers the opportunity to compare performance to an averaged indicator rate, preferably made up by a selection of comparable organisations. The stakeholders with reservations towards comparing safety performance question the added value of the function for two reasons: performance of different organisations is difficult to compare and the results can be misused.

For the first reason the example of "unstable approach rate" is often used. Each operator uses different approach criteria (e.g. different heights at which the aircraft needs to be stabilised), making comparison of unstable approach rates as determined by the operators meaningless. When the risk observatory has access to raw flight data, comparisons can be made using equal unstable approach criteria. However, pilots will act according to their criteria, making it more unlikely that an aircraft is stabilised at 1000 feet, when the criterion is 500 feet, compared to pilots operating with a criterion of 1000 feet. The latter concern is for higher management to misuse performance comparisons. In a world where resources are scarce, management can use safety performance comparisons to refrain from investing in safety. There may be a corporate view that disproportionate resources are being employed that might be better spent elsewhere, resulting in a temptation to save costs as the safety performance is acceptable compared to competition.

Even though there are reservations towards the idea of comparing safety information, it is an 
opportunity that cannot be dismissed. Safety analysts are needed to interpret the information to assure it is used in a proper manner: to increase the understanding of current safety performance and the possibilities for improvement.

\subsubsection{Provide qualitative safety data}

A clear - and perhaps surprising - need for most stakeholders is access to qualitative data. This includes hazards as identified by other organisations, for example to be used by an airline that starts operating to a new destination. Best practices and risk mitigating actions of other organisations could also be included.

A challenge for the risk observatory is to assure proper contextual information is available to the user in order to properly interpret the safety data. However, an operator can never obtain full access to all contextual information of another organisation; its operation, operating conditions, operating procedures, etc. Providing context to data shared in the risk observatory can only be done by the operator from which the data originates. For others it is impossible to contact the involved flight crews to get the proper context. Therefore, in the opinion of one consulted airline the processing, interpretation, validation and analysis of data should be done by the operator itself as it requires contextual data. These activities cannot be "outsourced" to a third party. The outcomes of this process are qualitative safety data, like safety reports and studies, best practices and hazards. These data are valuable to share.

\subsubsection{Provide the means to estimate the impact of safety measures}

An important part of safety management is to derive safety measures to reduce assessed risk. To close the management loop it is necessary to determine if these safety measures have the anticipated effect. It is therefore worthwhile to estimate that effect before implementing safety measures. This also facilitates comparing different measures to prioritise implementation based on impact on risk.

\section{The building blocks of the risk observatory}

Figure 3 presents the generic building blocks of the risk observatory.

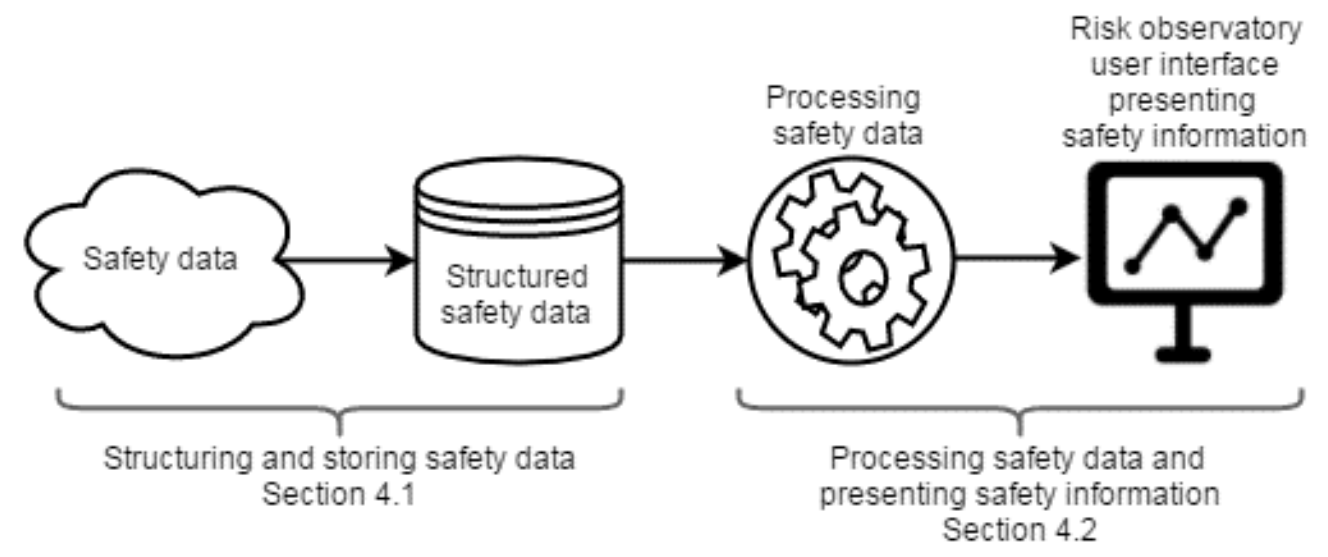

Figure 3. A generic overview of the risk observatory. 


\subsection{Structuring and storing safety data}

The available safety data needs to be accessed, structured and stored. This includes qualitative safety data as requested by the consulted stakeholders. Properly structuring the safety data makes it possible to combine data sources from a different nature (e.g. occurrence data and safety data) and allows safety performance comparison after the data is processed. The availability of recent, reliable and consistent data is essential to the proper functioning of the risk observatory. The usefulness of the risk observatory will be limited if data quality is poor. Organisations will need incentives to share data. An important incentive is that the organisations get useful safety information in return. The challenge is to assure that the risk observatory has access to proper data and functions as designed right from the start. Organisations sharing data will lose interested if this is not the case, further harming the viability of the risk observatory.

\subsection{Processing of safety data and presenting safety information}

There are two ways in which safety data is processed into safety information:

1. The safety data is directly visualised to improve the informative nature of the data.

2. The safety data is used to estimate risk using risk modelling. The risk is visualised.

\subsubsection{Visualise safety data}

Added value can be provided to safety data by processing and visualising the data such that it can be presented to a user as safety information. An example (for explanatory purposes, no real data used) is given in Figure 4 in which loss of separation events are plotted on a map of Europe. In this case safety data (loss of separations deducted from occurrence and/or radar data) are translated into safety information by plotting it on a map. In this way proper context is added to the input safety data.

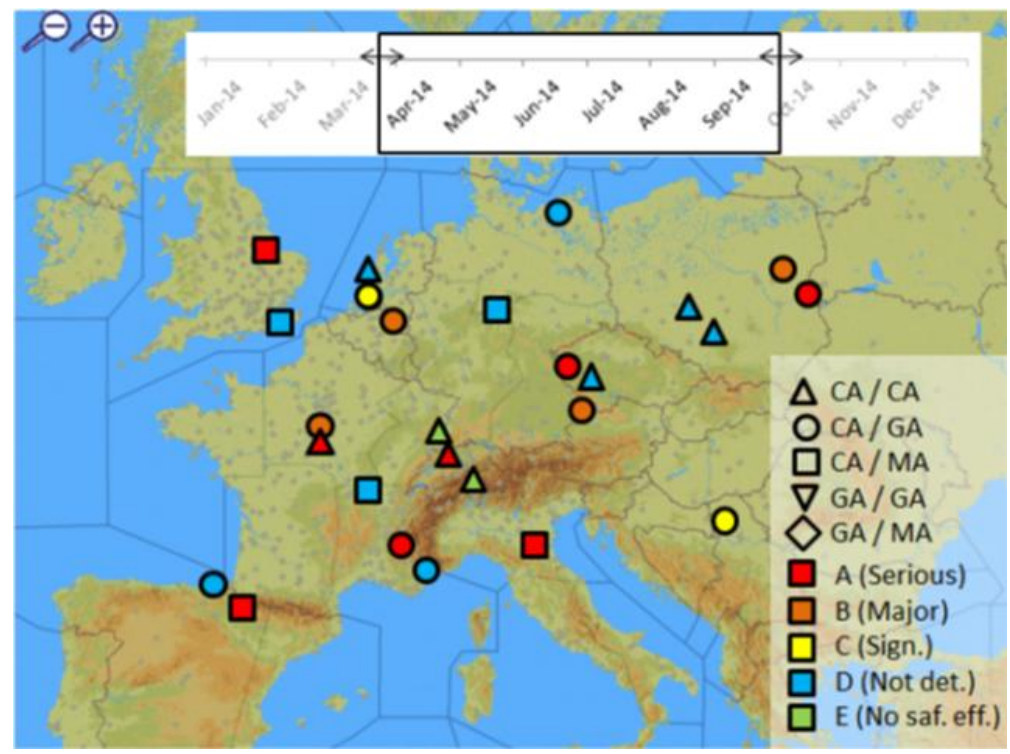

Figure 4. Geographical visualisation of loss of separation events (CA: commercial aviation, GA: general aviation, MA: military aviation) 


\section{MInstitute"}

\subsubsection{Visualise risk}

An important element of the risk observatory is the use of risk modelling to translate the input safety data into a picture of risk. To better explain the concept of visualising risk, use is made of the Causal Model for Air Transport (CATS) co-developed by NLR for the Dutch government (Ale, 2009). The causal risk model consists of generic accident scenarios in the form of Event Sequence Diagrams (ESDs). The ESDs show the quantified relation between a precursor and an accident. For the example used here, two precursors to a runway excursion are considered: an unstable approach and an inappropriate flare.

Figure 5 shows the monthly trend over a year of unstable approaches and inappropriate flares for a fictional organisation. These trends can be deduced from occurrence reports and/or flight data monitoring data and show the number of occurrences of these events per month over a year.
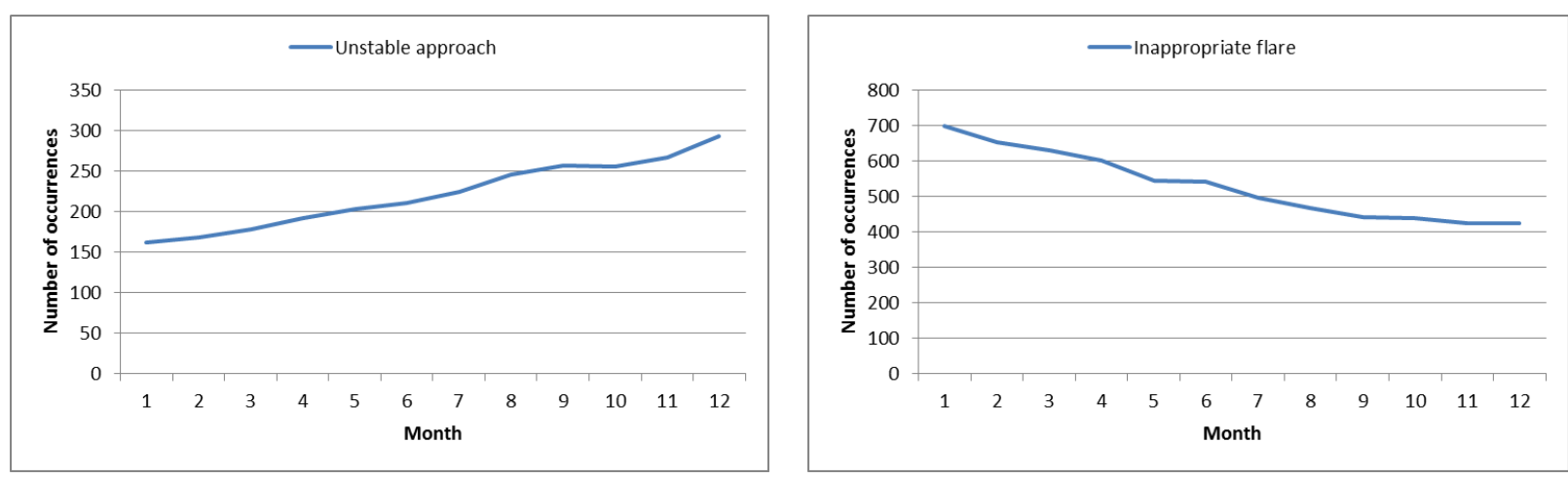

Figure 5. Monthly trends of unstable approaches and inappropriate flares (different y-axes).

Figure 6 shows a picture of the normalised runway excursion risk (value is 1.00 in month 1) per month over a year by processing the safety data using the risk model ${ }^{2}$. The plot also shows the relative influence of the two precursor types on the risk. Here it becomes clear that the rise in unstable approaches significantly increases runway excursion risk levels, outweighing the decrease in inappropriate flares. In this case safety data (unstable approaches and inappropriate flares deducted from occurrence and/or FDM data) are translated into safety information by estimated the associated risk using a risk model (it is noted that this example is given for explanatory purposes. For this specific case the cause-and-effect relationship between precursor and accident is not necessarily proven). This allows resources to be used for actions that have the biggest impact on risk.

\footnotetext{
2 The runway excursions considered are of accident severity (runway excursions can be less severe). Only runway excursions are considered that take place after either an unstable approach or an inappropriate flare occurred.
} 


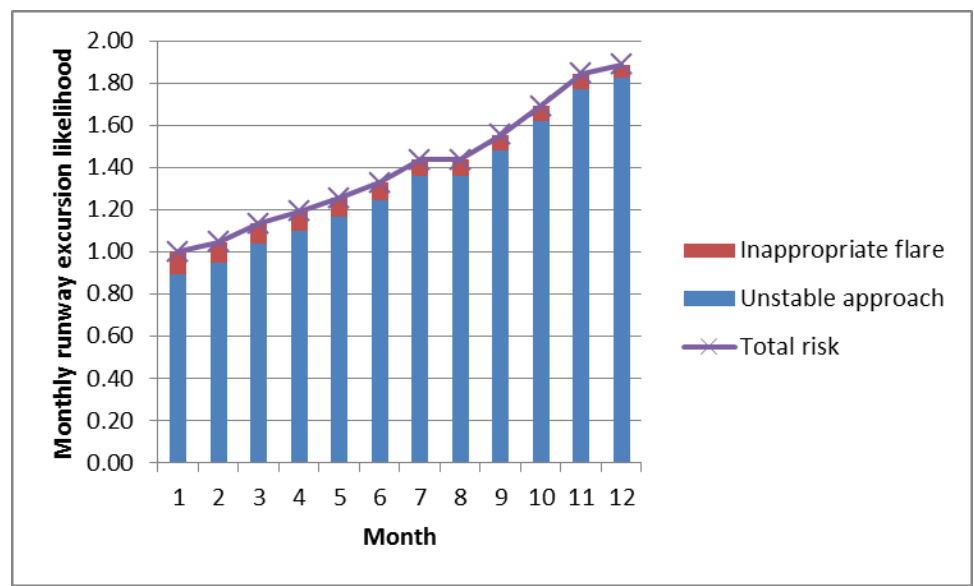

Figure 6. Runway excursion risk due to unstable approaches and inappropriate flares.

\section{The early prototype of the risk observatory}

In the first year of the project a design-focused approach was followed by developing an early prototype. The early prototype is a mock-up demonstrating the end-user experience. The design of the early prototype was conducted iteratively by the project team using the requirements drafted from stakeholder consultations, literature research and consortium expertise. The early prototype is used to validate the requirements with the stakeholders. This is done by demonstrating and discussing the early prototype with a selection of stakeholders that were consulted in order to elicit the requirements.

The early prototype has four main dashboards and a homepage that gives access to the dashboards. The four dashboards are: (1) an occurrences dashboard, (2) a risk dashboard, (3) a search dashboard and (4) a what-if analysis dashboard. The project team used the software tool Balsamiq to implement the early prototype in the form of a mock-up of web-based dashboards. Balsamiq enables building website blueprints, which presents the visual aspects and interaction of a user with a website.

\subsection{The login page and homepage}

Figure 7 shows the login to enter the risk observatory. Depending on the authorisation or user profile, the user will enter a homepage that is tailored to the specific user's domain with relevant safety performance indicators (SPI) and associated risks. Figure 8 shows a screenshot of the homepage showing the noticeable trends in SPI and risks (coloured arrows). This way the user is directly pointed to areas of concern, a clear wish from consulted stakeholders. By clicking on the indicator or accident type the user can directly drill down into the underlying safety data and trend analyses. The homepage also provides access to the search dashboard and what-if analysis dashboard. 


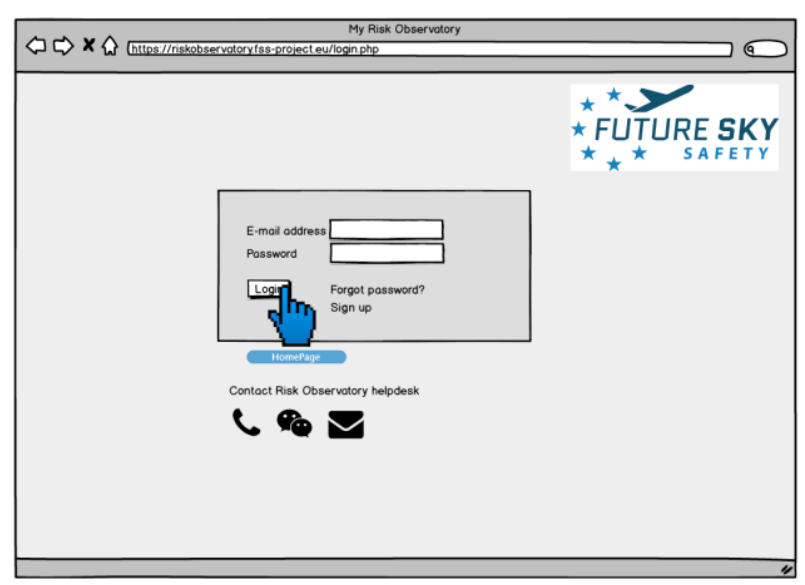

Figure 7. Login page.

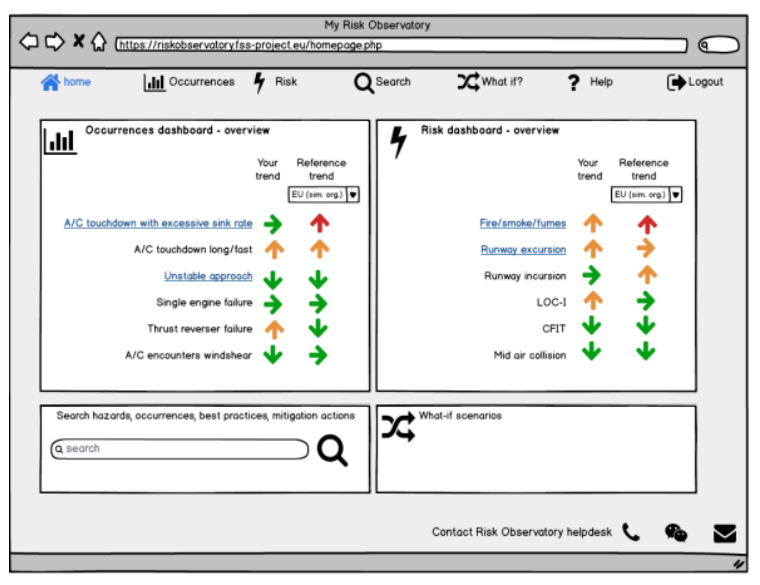

Figure 8. Homepage.

\subsection{The occurrence and risk dashboard}

Figure 9 shows the occurrence dashboard, where the user can monitor the number of occurrences or rate of a particular SPI. There are several functionalities available to the user, for example filtering settings, ability to access the underlying data or link to the risk dashboard to view the risk associated with the occurrence type. It is also possible to compare performance against a relevant average, a need derived from stakeholder consultations as indicated in Section 3.2.1. The occurrence dashboard presents information directly derived from reported occurrences and measured events. It corresponds to the first method in which the risk observatory translates safety data into safety information (see Section 4.2).

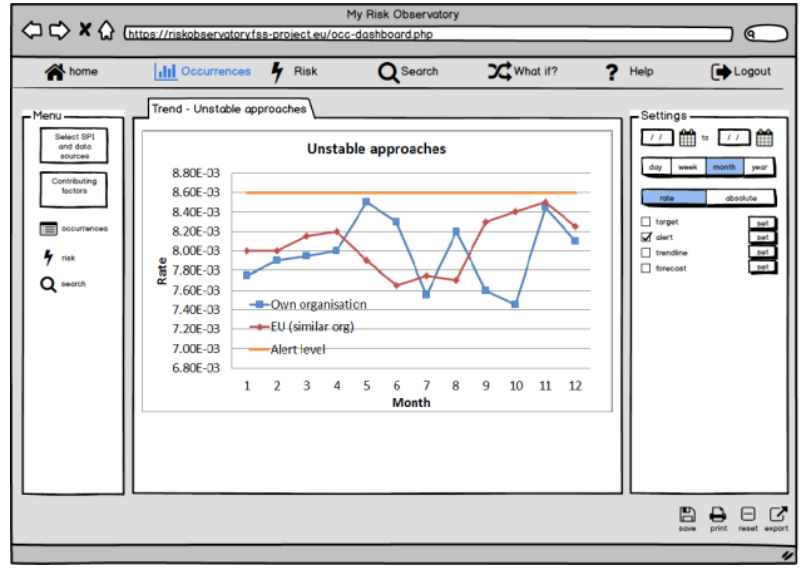

Figure 9. The occurrence dashboard.

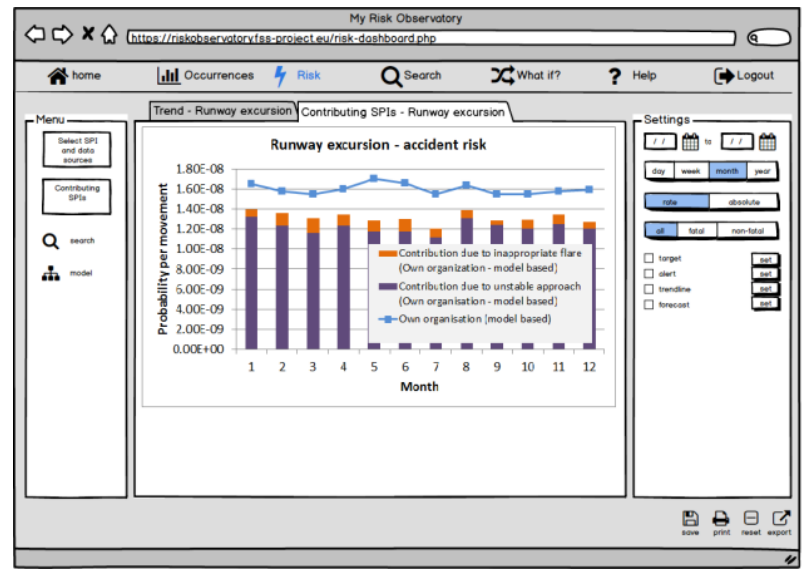

Figure 10. The risk dashboard.

Figure 10 shows the risk dashboard. On this page the user can observe the trend in accident risk for a particular accident type for their own organisation, and compare that against a benchmark risk level and a user defined alert level. The information shown on the dashboard is derived from combining reported occurrences and measured events with risk models to estimate risk. It corresponds to the second method in which the risk observatory translates safety data into safety information (see Section 4.2). 


\subsection{The search dashboard}

Figure 11 shows a screenshot of a dashboard that can be used to search for hazards, occurrences, best practices and mitigation actions stored in a database in the risk observatory. The idea is that stakeholders share such information, to have it available to other organisations through the search dashboard. The inclusion of this functionality is a clear wish from the stakeholders consulted, as stated in Section 3.2.2. The search dashboard can be extended in functionality by including shared safety analyses and safety reports from stakeholders.

\subsection{The what-if analysis dashboard}

Figure 12 shows the what-if analysis dashboard where the user can perform comparative analysis by altering SPI rates to estimate the effect on risk. The user can select SPIs and associated risks (accident types) and assess the impact on the risk level of changing the frequency of occurrence of the SPIs. This allows improved decision making: a user can determine which SPI frequency reduction has the largest impact on risk. Resources can then be used for the biggest opportunity for risk mitigation. The what-if analysis functionality makes use of risk models and is a clear wish from stakeholders, as indicated in Section 3.2.3.

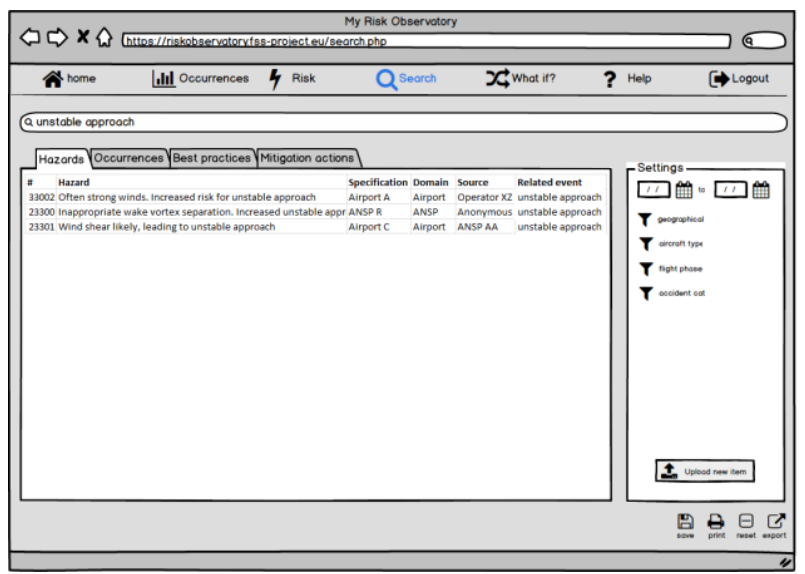

Figure 11. The search dashboard.

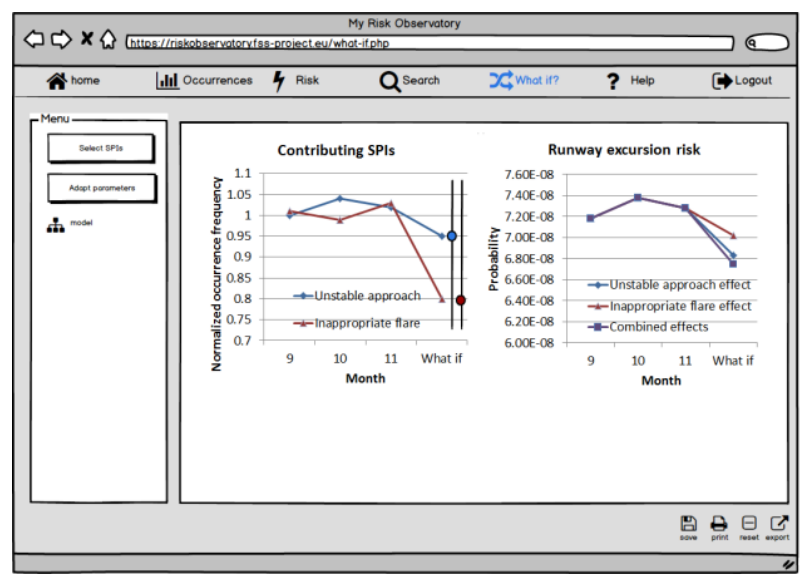

Figure 12. The what-if dashboard.

\section{How does the risk observatory provide the needed breakthrough?}

The question arises what the risk observatory is going to offer towards reaching the goal of 1 accident per 10000000 flights. The strength of proper safety management is that it can influence safety performance throughout the system. Meaning it can mitigate risk coming from technical failures, organisational failures, human factors and environmental influences.

The key added value of the risk observatory is its provision of access to safety information across organisations and across domains. It is foreseen that this has four effects that can boost safety performance.

\subsection{More safety information available to individual organisations}

Judging if your safety performance is acceptable can be a challenge, especially for smaller 
operators. This makes comparing of performance to a relevant average safety performance a valuable function, since it provides additional information that can be used in the judgment. Sharing qualitative safety data such as hazards, best practices and mitigations is also of added value to safety practitioners.

\subsection{Support business cases for mitigating overarching risks}

The risk observatory allows identifying hazards and mitigating risks that are shared or common to different organisations and/or domains. This way consensus and momentum can be built behind those actions that will make the greatest difference in risk but cannot be realised by individual organisations. Business cases of safety actions that go beyond the reach (financially, organisationally, politically) of individual organisations can be made.

\subsection{Focus resources on highest risks and/or risk with highest opportunity for mitigation}

Risk modelling allows you to make your safety management effort risk based. If you are able to estimate risk levels, and estimate the impact of mitigations on risk level, you are able to spend your resources such that the highest possible risk reduction per euro spend are achieved. This is preferable to ad-hoc event-driven safety management.

\subsection{Tackle concerns at interfaces between organisations and domains}

Risks at interfaces between organisations and domains are currently a blind spot for most safety management systems. By offering and combining data from different organisations and different domains these risk can be identified, assessed and mitigated if needed.

\section{Conclusion}

The project team received a positive response from foreseen users on the demonstrated functionalities and design of the early prototype. The design-focused approach taken with developing the early prototype helped in eliciting feedback on the user requirements from stakeholders. The risk observatory should provide safety information to its users to trigger further analysis and mitigation of areas with increased risk. The risk observatory should provide qualitative and quantitative safety information. Key areas of further research are data availability, reliability and protection, the validation and verification of the risk models applied in the risk observatory and methods to ensure that contextual information can be maintained during data fusion and made available in the risk observatory.

\section{Acknowledgement}

This project has received funding from the European Union's Horizon 2020 research and innovation programme under grant agreement No 640597.

\section{References}

Ale, Ben et al. (2009, March). Causal Model for Air Transport Safety. Final report

CICTT (2013). Aviation Occurrence Categories. Definitions and Usage Notes. Retrieved from http://www.intlaviationstandards.org/Documents/OccurrenceCategoryDefinitions.pdf 
EASA (2015). Annual Safety Review 2014. Retrieved from

https://www.easa.europa.eu/document-library/general-publications/

European Commission (2011). Flightpath 2050 Europe's Vision for Aviation. Retrieved from http://ec.europa.eu/transport/modes/air/doc/flightpath2050.pdf

FAA (2016). FAA Aviation Safety Information Analysis and Sharing. Retrieved from www.asias.faa.gov/

Hoffmann, Thomas (2016). How an ANSP monitors and manages safety threats - seeing around the corner. Retrieved from http://www.optics-project.eu/?page id=877

IAEA (2008). The IAEA/NEA Incident Reporting System (IRS). Retrieved from https://www-ns.iaea.org/downloads/ni/irs/iaea-nea-irs2008.pdf

ICAO (2001). Annex 13 to the Convention on International Civil Aviation. Aircraft Accident and Incident Investigation. Ninth Edition

ICAO (2013). 2014 - 2016 Global Aviation Safety Plan. Retrieved from http://www.icao.int/publications/Pages/Publication.aspx?docnum=10004

ICAO (2016). Annex 19 to the Convention on International Civil Aviation. Safety Management. Second Edition

Roelen, A.L.C. et al. (2008). Quantification of Event Sequence Diagrams for a causal risk model of commercial air transport. Report no. NLR-CR-2008-648

\section{Glossary}

Accident: An occurrence associated with the operation of an aircraft, which takes place between the time any person boards the aircraft with the intention of flight until such time as all such persons have disembarked, in which: (a) a person suffers a fatal or serious injury, (b) the aircraft sustains damage or structural failure, or (c) the aircraft is missing or is completely inaccessible. For the full definition please refer to ICAO Annex 13 (ICAO, 2001).

Inappropriate flare: a flare (a manoeuvre by the pilot that reduces the rate of descent so that an excessively hard touchdown is avoided) that starts from a stabilised condition at the runway threshold but the manoeuvre itself is conducted inappropriately (Roelen et al., 2008).

Runway excursion: A veer off or overrun off the runway surface (CICTT, 2013).

Safety data: facts or figures derived from sources such as occurrence reports and FDM programmes. For example, the number of unstable approaches or loss of separation events in a period. Safety data is rarely useful by itself until it is processed and organised in a specific context, which then becomes safety information.

Safety information: safety data organised and processed in a specific context, allowing the recipient of the information to make decisions on future actions. Example: "a significant reduction in the rate of unstable approaches for runway 99 at XYZ after a crew memo was issued reminding crews about the established standard operating procedure." 


\section{Macrothink

Unstable approach: an approach is considered unstable if specific criteria are not met during the approach to land. The specific criteria are included in (Roelen et al., 2008).

\section{Copyright Disclaimer}

Copyright for this article is retained by the author(s), with first publication rights granted to the journal.

This is an open-access article distributed under the terms and conditions of the Creative Commons Attribution license (http://creativecommons.org/licenses/by/3.0/). 